\title{
THE STRUCTURE OF THE LOCAL HOT BUBBLE
}

\author{
W. Liu ${ }^{1}$, M. ChiaO ${ }^{2}$, M. R. Collier ${ }^{2}$, T. Cravens $^{3}$, M. Galeazzi ${ }^{1}$, D. Koutroumpa ${ }^{4}$, K. D. Kuntz ${ }^{5}$, R. Lallement ${ }^{6}$,

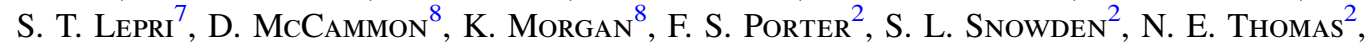 \\ Y. UPRETY ${ }^{1,10}$, E. URSINO ${ }^{1,11}$, AND B. M. WALSH ${ }^{9}$ \\ ${ }^{1}$ Department of Physics, University of Miami, Coral Gables, FL, 33124, USA; galeazzi@physics.miami.edu \\ ${ }^{2}$ NASA Goddard Space Flight Center, Greenbelt, MD, 20771, USA \\ ${ }^{3}$ Department of Physics and Astronomy, University of Kansas, Lawrence, KS 66045, USA \\ ${ }^{4}$ Universite Versailles St-Quentin; Sorbonne Universites, UPMC Univ. Paris 06 \& CNRS/INSU, LATMOS-IPSL, F-78280, France \\ ${ }^{5}$ The Henry A. Rowland Department of Physics and Astronomy, Johns Hopkins University, Baltimore, MD 21218, USA \\ ${ }^{6}$ GEPI Observatoire de Paris, CNRS, Universite Paris Diderot, F-92190, Meudon, France \\ ${ }^{7}$ Department of Atmospheric, Oceanic, and Space Sciences, University of Michigan, Ann Arbor, MI 48109, USA \\ ${ }^{8}$ Department of Physics, University of Wisconsin, Madison, WI 53706, USA \\ ${ }^{9}$ Department of Mechanical Engineering, Boston University, Boston, MA 02215, USA \\ Received 2016 September 12; revised 2016 November 2; accepted 2016 November 5; published 2016 December 28
}

\begin{abstract}
Diffuse X-rays from the Local Galaxy (DXL) is a sounding rocket mission designed to quantify and characterize the contribution of Solar Wind Charge eXchange (SWCX) to the Diffuse X-ray Background and study the properties of the Local Hot Bubble (LHB). Based on the results from the DXL mission, we quantified and removed the contribution of SWCX to the diffuse X-ray background measured by the ROSAT All Sky Survey. The "cleaned" maps were used to investigate the physical properties of the LHB. Assuming thermal ionization equilibrium, we measured a highly uniform temperature distributed around $k T=0.097 \mathrm{keV} \pm 0.013 \mathrm{keV}(\mathrm{FWHM}) \pm 0.006 \mathrm{keV}$ (systematic). We also generated a thermal emission measure map and used it to characterize the three-dimensional (3D) structure of the LHB, which we found to be in good agreement with the structure of the local cavity measured from dust and gas.
\end{abstract}

Key words: ISM: bubbles - ISM: structure - X-rays: diffuse background - X-rays: ISM

\section{INTRODUCTION}

The diffuse soft X-ray background observed at $1 / 4 \mathrm{keV}$ in the ROSAT R12 band (Snowden et al. 1997) is dominated by a local source that shows no sign of absorption by cool interstellar gas (Juda et al. 1991). One optical depth at 1/

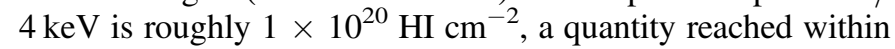
$50 \mathrm{pc}$ at average interstellar densities. An irregular "local cavity" extending about $100 \mathrm{pc}$ from the Sun was shown by the Copernicus satellite to be almost entirely devoid of cool gas (Savage \& Jenkins 1972; Jenkins \& Meloy 1974; Knapp 1975). If filled with $10^{6} \mathrm{~K}$ gas at a reasonable pressure, the cavity could produce observed "local" X-rays (Sanders et al. 1977). The portion of the local cavity filled with this hot gas was dubbed the Local Hot Bubble (LHB) (Sanders et al. 1977; Tanaka \& Bleeker 1977; Cox \& Snowden 1986), and the enhanced X-ray emitting areas at intermediate latitudes were found to correlate well with minima in the measured neutral gas column (Snowden et al. 1990), as if the cool gas had been displaced by the hot gas. ROSAT demonstrated that a smaller portion of the soft X-ray background is due to the Galactic halo (Burrows \& Mendenhall 1991; Snowden et al. 1991). Emission from the hot Galactic halo contributes significantly only in areas of low absorption at intermediate and high Galactic latitudes.

This simple picture was upset by the discovery of diffuse $\mathrm{X}$-ray emission from within the solar system, due to Solar Wind Charge eXchange (SWCX), which could provide some

\footnotetext{
${ }^{10}$ Current address: Department of Physics and Astronomy, Middle Tennessee State University, Murfreesboro, TN 37132, USA.

${ }^{11}$ Current address: Physics Department, Grinnell College, Grinnell, IA 50112, USA.
}

or all the soft diffuse X-ray emission at $1 / 4 \mathrm{keV}$ (Cravens 2000; Cravens et al. 2001; Robertson \& Cravens 2003; Lallement 2004; Koutroumpa et al. 2009). SWCX emission is generated when the highly charged solar wind ions interact with the neutral materials within the solar system, gaining an electron in a highly excited state which then decays emitting an $\mathrm{X}$-ray photon with the characteristic energy of the ion. In order to improve our understanding of the local diffuse X-ray emission and the structure of the LHB, it is essential to remove the contamination of the SWCX. However, despite many efforts, an accurate estimation of the SWCX is quite difficult, especially in the $1 / 4 \mathrm{keV}$ band, due to the poorly known cross sections for producing the many X-ray lines from SWCX, limited data on heavy ion fluxes in the Solar Wind, and the general spectral similarity of SWCX and thermal emission (Cravens 2000; Lallement 2004; Koutroumpa et al. 2006; Henley \& Shelton 2008; Snowden et al. 2009; Yoshino et al. 2009; Crowder et al. 2012). Efforts to estimate the SWCX contribution to historical measurements for the diffuse X-ray background, such as in the ROSAT All-Sky Survey (RASS) are even more problematic due to the limited solar wind data (see Kuntz et al. 2015, for further discussion).

Diffuse X-rays from the Local Galaxy (DXL) (Galeazzi et al. 2011, 2012; Thomas et al. 2013) is a sounding rocket mission designed to quantify and characterize the contribution of SWCX to the diffuse X-ray emission. To separate the SWCX contribution, $D X L$ uses the spatial signature of SWCX emission due to the "helium focusing cone," a higher neutral He density region downwind of the Sun (Michels et al. 2002; Snowden et al. 2009; Thomas et al. 2013). By comparing the $D X L$ data and the RASS data along the DXL scan path, our team measured the broad band averaged cross sections and provided 

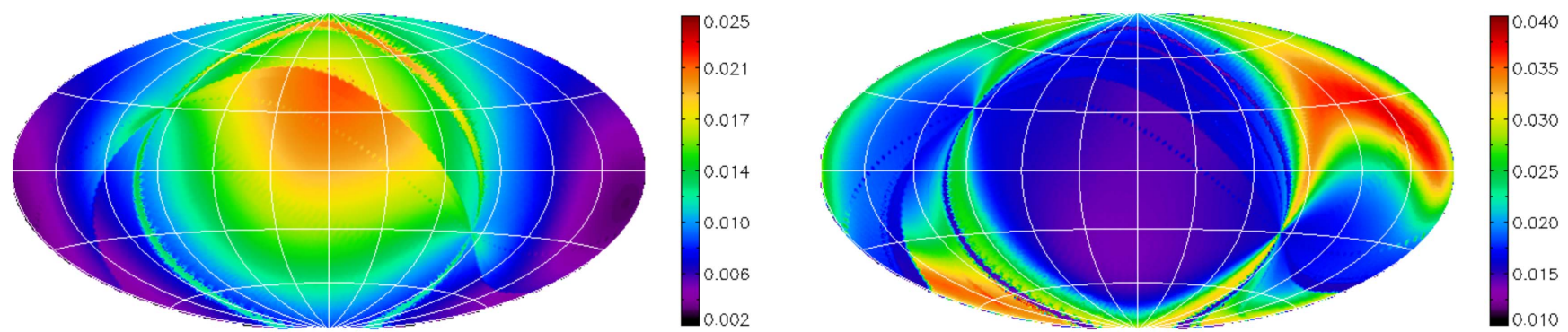

Figure 1. The Aitoff-Hammer projection of the neutral integral distribution for $\mathrm{H}$ (left) and He (right) in units of $\mathrm{cm}^{-3} \mathrm{au}^{-1}$.

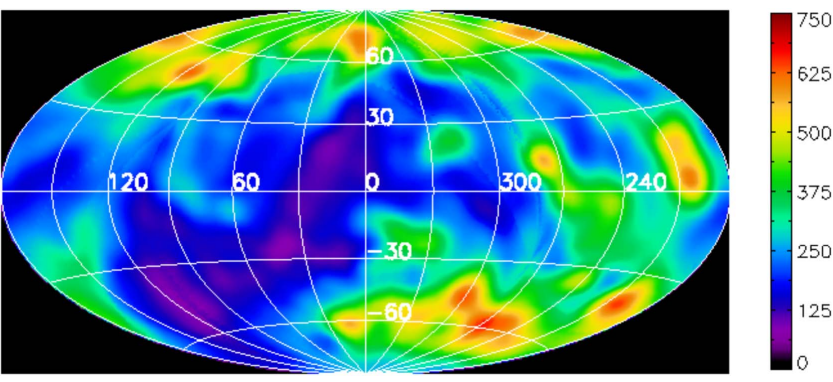

Figure 2. The Aitoff-Hammer projection of the total "cleaned" LHB emission in the RASS R1+R2 band in RU after removing both the non-local components and SWCX contribution.

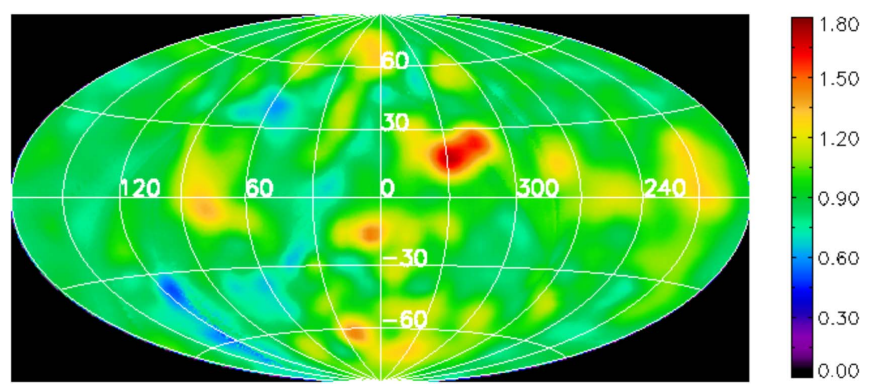

Figure 3. The Aitoff-Hammer projection of the R2/R1 band ratio of the "cleaned" LHB.

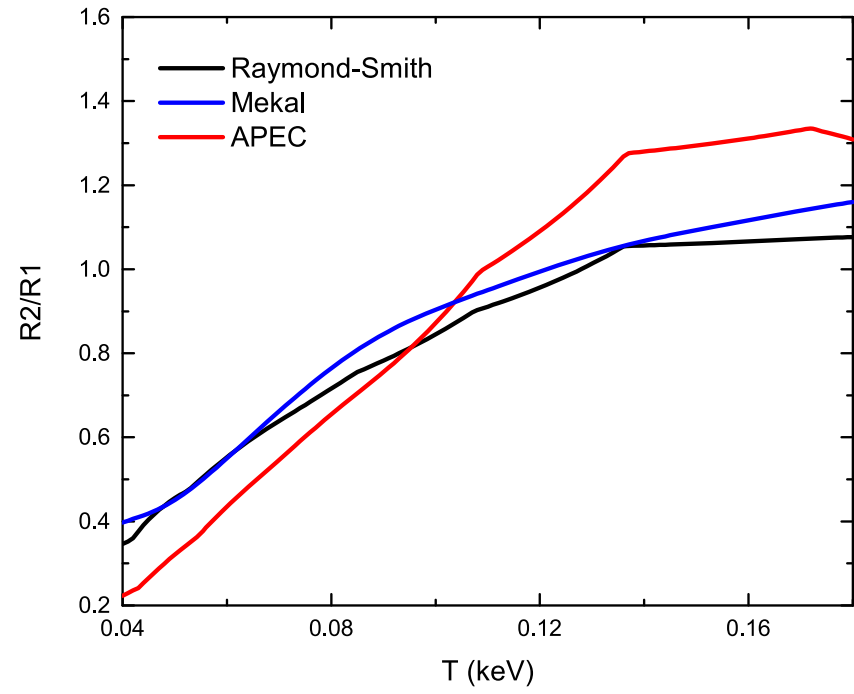

Figure 4. R2/R1 ratio as a function of temperature from the Raymond-Smith model (black), Mekal model (blue), and APEC model (red) with Anders \& Grevesse (1989) abundance table.

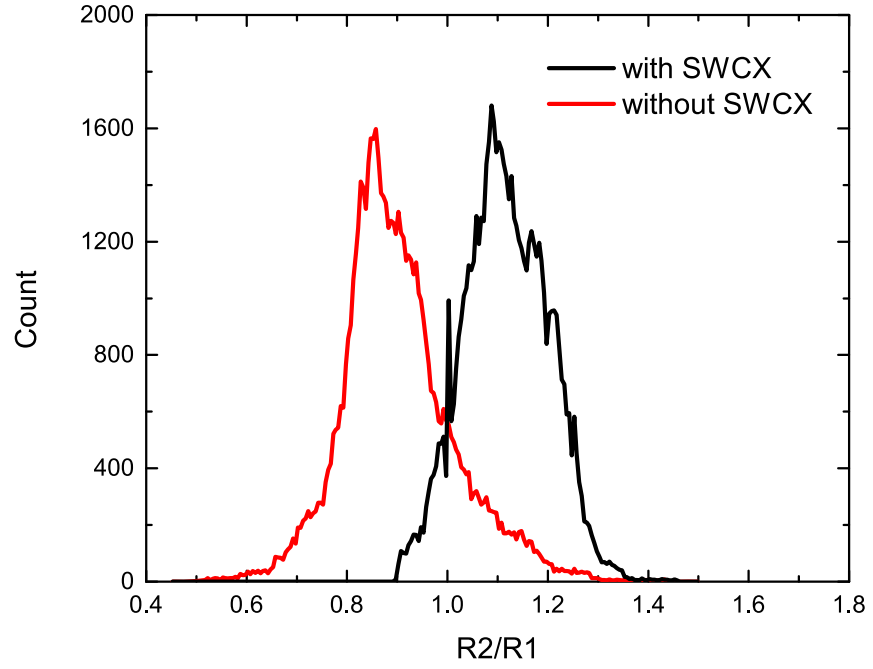

Figure 5. The distribution of R2/R1 ratio before subtracting the SWCX (in black) and after subtracting the SWCX (in red).

a significantly more accurate empirical estimate of the SWCX emission. DXL estimated the total SWCX contribution to be $\sim 40 \%$ of the X-ray flux at $1 / 4 \mathrm{keV}$ in the Galactic plane (Galeazzi et al. 2014), supporting the previous picture of a hot bubble filling the local interstellar medium in all directions and accounting for the remaining $\sim 60 \%$ in the plane. Based on the results from Galeazzi et al. (2014), Snowden et al. (2014) showed that the gas pressure from the remaining local emission is in pressure equilibrium with the local interstellar clouds, eliminating the long standing pressure problem of the LHB (Jenkins 2009).

In this paper we re-evaluate the properties of the LHB based on the RASS data (Snowden et al. 1997) combined with the estimate of the SWCX contribution from $D X L$. We focused on the R1 ( 0.11-0.284 keV) and R2 ( 0.14-0.284 keV) data, as the LHB contribution to the R4 $(\sim 0.44-1.01 \mathrm{keV})$ and R5 $(\sim 0.56-1.21 \mathrm{keV})$ bands is negligible. In Section 2 we describe how to remove the SWCX emission from the RASS data, and to estimate the LHB temperature and emission measure, Section 3 contains the results, and conclusions are in Section 4.

\section{DATA ANALYSIS}

Snowden et al. (1998, 2000) used the shadows cast by nearby $(100-200 \mathrm{pc})$ clouds to estimate and remove the contribution from background emission (Galactic halo and extragalactic components) to the RASS R1 and R2 maps, producing clean "local" maps $(<100-200 \mathrm{pc})$. With the advance in X-ray telescopes, the shadow technique is now 

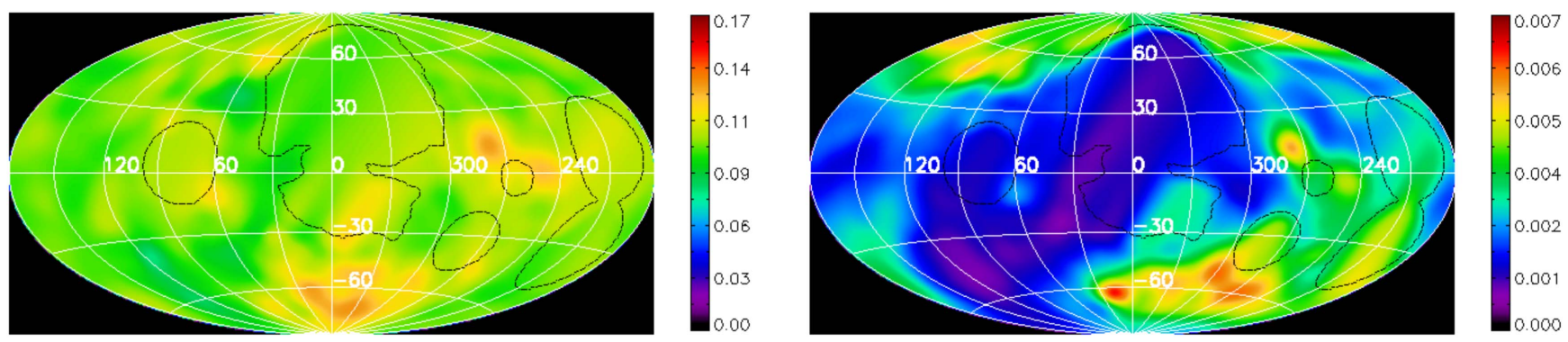

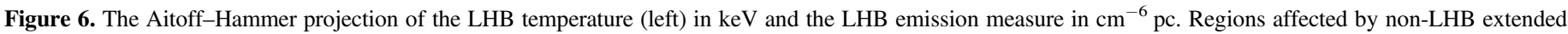
sources have been interpolated and circled in black.

feasible for individual pointing for spectroscopy study to disentangle the foreground and background (Galeazzi et al. 2007; Smith et al. 2007; Gupta et al. 2009; Henley et al. 2015; Liu et al. 2016; Ursino et al. 2016). These "local" maps should contain only the contribution from SWCX, both heliospheric $(S(\ell, b, t))$ and geocoronal $(G)$, and the LHB $(L(\ell, b))$. For each RASS band we can therefore write the total flux, $F(\ell, b)$, as:

$$
F(\ell, b, t)=S(\ell, b, t)+L(\ell, b)+G
$$

Following the procedure from Uprety et al. (2016), based on the models of Koutroumpa et al. (2006), the heliospheric component can be written as

$$
S(\ell, b, t)=\beta(t) \times N(\ell, b)
$$

where

$$
\beta(t)=n_{p}\left(R_{0}, t\right) v_{\text {rel }}(t) \alpha_{\mathrm{He}}
$$

depends on the solar wind properties and the cross section with neutrals $\left(n_{p}\left(R_{0}, t\right)\right.$ is the proton density at $R_{0}=1 \mathrm{au}, v_{\text {rel }}$ is the relative speed between solar wind and neutral flow, and $\alpha_{\mathrm{He}}$ is the compound cross-section for Helium), and

$$
N(\ell, b)=\int \frac{n_{\mathrm{He}}}{R^{2}} d s+\frac{\alpha_{\mathrm{H}}}{\alpha_{\mathrm{He}}} \int \frac{n_{\mathrm{H}}}{R^{2}} d s
$$

where $\int \frac{n}{R^{2}} d s$ is the integrated neutral column density along the line of sight, weighted by one over the distance from the Sun squared, and $\frac{\alpha_{\mathrm{H}}}{\alpha_{\mathrm{He}}}$ is the ratio between cross sections with $\mathrm{H}$ and $\mathrm{He}$.

Uprety et al. (2016) combined data from the same part of the sky from $D X L$ and RASS and found the best fit parameter for $\beta(t)$ for each RASS band for given values of $G$ and $\alpha_{\mathrm{H}} / \alpha_{\mathrm{He}}$. Therefore, the heliospheric SWCX contribution to any RASS band for any direction can be directly estimated given the neutral distribution. Figure 1 shows the Aitoff-Hammer projection of the $\mathrm{H}$ and $\mathrm{He}$ neutral integral during the RASS campaign, calculated based on a well determined model for the interstellar neutral distributions within the solar system (Lallement et al. 1985a, 1985b; Lallement 2004; Koutroumpa et al. 2006). The sharp edges visible in this maps are due to abrupt shifts in vantage point around the Earth's orbit during the ROSAT survey, since the survey comes back to its starting point after six months and there were missed sections that were backfilled at later times.

Uprety et al. (2016) extensively discuss the use of different combinations of $G$ and $\alpha_{\mathrm{H}} / \alpha_{\mathrm{He}}$ and their effect of the systematic error of their results. For this investigation, we use their best fit parameters for $\beta(t)$ shown in their Table 2, combined with the neutral distribution shown in Figure 1 to generate maps of SWCX contribution to both R1 and R2. The model SWCX maps were then subtracted from the local maps of Snowden et al. $(1998,2000)$ to produce the "clean" LHB maps in the R1 and R2 band which contain only the LHB emission. We note that these maps are different from those shown in Uprety et al. (2016). Uprety et al. (2016) subtracted the SWCX contribution for the total R1 and R2 bands, producing "clean" astrophysical maps, containing both local and non-local components. Figure 2 shows the Aitoff-Hammer projection of the total LHB emission in $\mathrm{R} 1+\mathrm{R} 2$ band, and Figure 3 shows the projection of the R2/R1 ratio of the LHB (notice that all the maps are smoothed since the LHB emission is assumed to vary smoothly over large angular scales and our analysis is insensitive to any variation on finer scales).

Assuming that the LHB is in collisional equilibrium and can be well represented by a single, unabsorbed thermal component, it is possible to estimate the temperature of the LHB in any given direction based on the ratio of the R2/R1 bands. Unfortunately, none of the current thermal models available in $\mathrm{XSPEC}^{12}$ are particularly accurate in the R1 and R2 bands. The Raymond-Smith model (Raymond \& Smith 1977) estimates the emission of a large number of weak lines that are known to be present, but it lacks accurate excitation rates and wavelengths. The Mekal model (Mewe et al. 1985; Kaastra \& Mewe 1993) is identical in treatment of ionization balance with the Raymond-Smith model, but has many more lines and updated Fe L calculations. The APEC model (Smith et al. 2001) includes only transitions for which accurate atomic rates are available and lacks many lines at low energy. In Figure 4 we plot the R2/R1 ratio as a function of temperature using the Raymond-Smith, Mekal, and APEC models with Anders \& Grevesse (1989) abundance table. It is immediately evident that the curve for APEC model is quite different from the other two and, therefore, any conclusion will depend on the model used. However, as it turns out, the systematic effect introduced by the choice of model is not large as our LHB data, as we will show in the next section, are clustered in the region where the curves nearly overlap.

We also point out that in the RASS maps there are brighter regions associated with additional $\mathrm{X}$-ray emission from extended sources, e.g., the Cygnus Loop, Vela SNRs, the Galactic halo beyond the Draco Clouds, and the Monogem Ring. We have excluded them in our study, both by setting an upper limit in the RASS R4+R5 value, and manually removing regions associated with known structures unrelated to the LHB.

\footnotetext{
${ }^{12}$ http://heasarc.gsfc.nasa.gov/xanadu/xspec/
} 

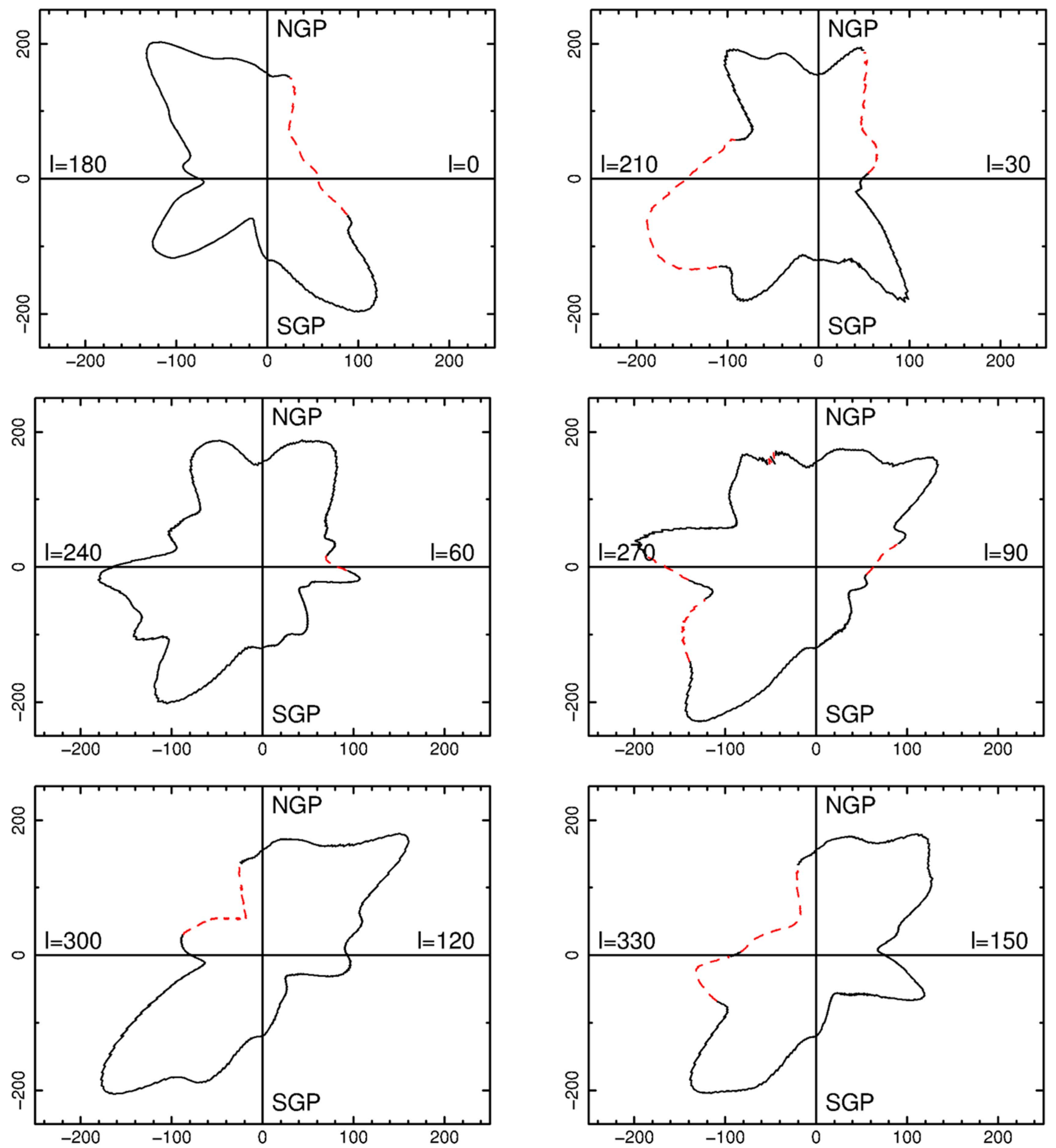

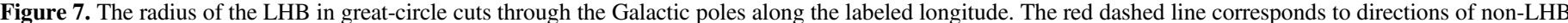
bright extended sources.

\section{RESULTS}

\subsection{The LHB Temperature}

In Figure 5 we show the distribution of the R2/R1 ratio over the whole sky before (in black) and after (in red) removing the SWCX contribution from the RASS data. The peak of R2/R1 is shifted from $\sim 1.09$ to 0.86 , with a FWHM of 0.16 . This corresponds to a temperature of $k T=0.099 \mathrm{keV}$ using the APEC model, $0.103 \mathrm{keV}$ for Raymond-Smith model and $0.091 \mathrm{keV}$ for Mekal model. The difference between APEC and Raymond-Smith models is very small since the R2/R1 ratio of 0.86 is in a region where the three lines in Figure 4 are very close to each other. Combining the three results, we estimate the peak temperature as $k T=0.097 \mathrm{keV}$, with a systematic error of $0.006 \mathrm{keV}$. This systematic error also includes the systematic uncertainties in the SWCX parameters discussed in Uprety et al. (2016). Based on the the relation between the $\mathrm{R} 2 / \mathrm{R} 1$, the temperature of the LHB is therefore cooler than previous estimates based on the maps without SWCX subtraction (Snowden et al. 1998; Kuntz \& Snowden 
Galactic Plane

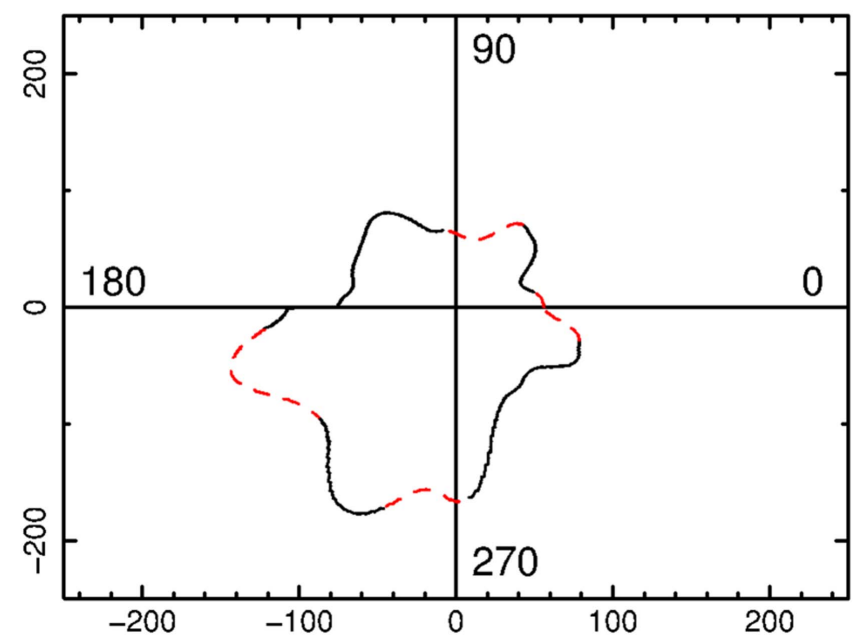

Figure 8. The radius of the LHB in great-circle cuts through the Galactic plane. The red dashed line corresponds to directions of non-LHB bright extended sources.

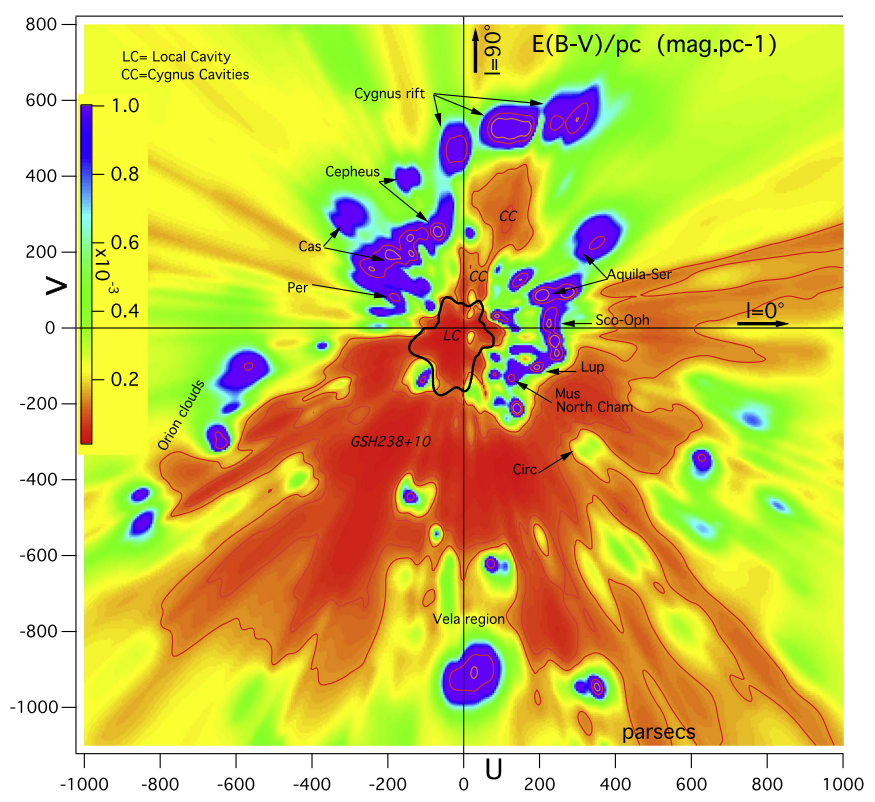

Figure 9. Differential color excess shows the inverted differential opacity distribution in the Galactic plane (map is taken from Lallement et al. 2014). The black line shows the contour of the LHB in the Galactic plane from our measurements.

2000). In the left of Figure 6, we also show the Aitoff-Hammer projection of the temperature of the LHB. The distribution of the LHB temperature is quite uniform with a FWHM of $0.013 \mathrm{keV}$. In the figure, the areas affected by non-LHB extended sources have been interpolated and circled in black.

\subsection{The LHB Emission Measure and Size}

We also used the new R1 and R2 maps to extract the emission measure (EM) of the emitting plasma for each direction in the sky based on the APEC model. The AitoffHammer projection of the LHB EM is shown on the right of Figure 6. The EM is generally larger toward high latitude while smaller at low latitude in the northern hemisphere. In the southern hemisphere, the EM is small from $0^{\circ}<1<180^{\circ}$. It is

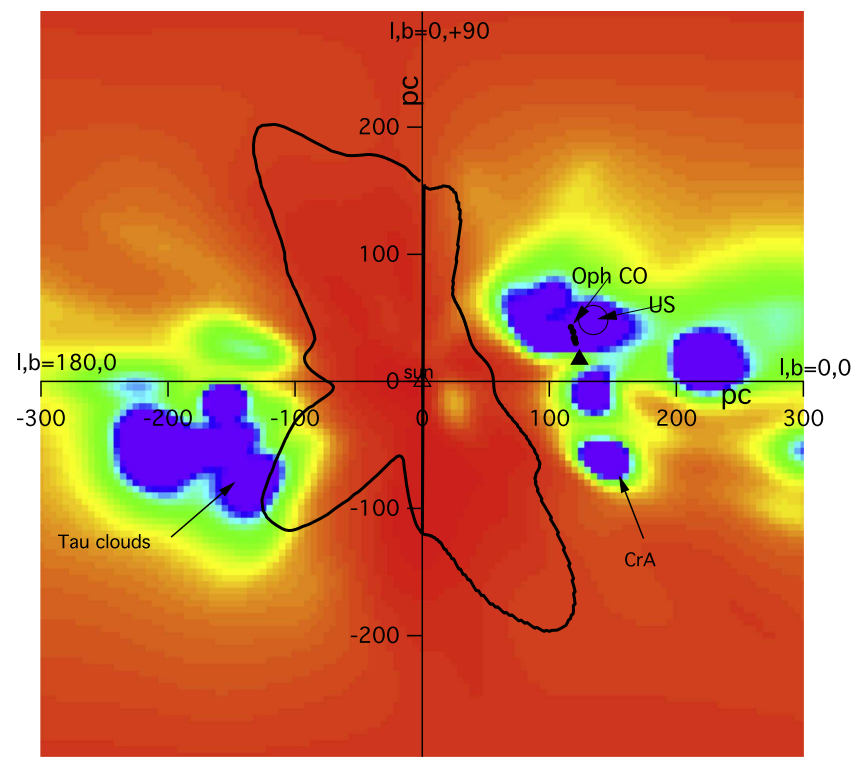

Figure 10. Same as Figure 9 but in the vertical plane and on a smaller scale.

small at low latitude and large at high latitude from $180^{\circ}<1<$ $360^{\circ}$. Over the whole sky, the distribution ranges from $\sim 0.8 \times 10^{-3} \mathrm{~cm}^{-6} \mathrm{pc}$ to $\sim 6.5 \times 10^{-3} \mathrm{~cm}^{-6} \mathrm{pc}$.

Assuming that the electron density in the LHB is constant, we can use the EM to estimate the size of the LHB. For constant electron density, the $\mathrm{EM}$ is expressed as $\mathrm{EM}=n_{e} n_{p} L$, where $n_{e}$ and $n_{p}$ are the electron and proton densities, $L$ is the path length through the LHB emitting plasma. Adopting the electron value of $n_{e}=4.68 \times 10^{-3} \mathrm{~cm}^{-3}$ (Snowden et al. 2014), we estimated the extension of the LHB in all directions and we built its three-dimensional structure. Figures 7 and 8 show the extension of the LHB along great-circle cuts through the Galactic pole and Galactic plane. The dash lines correspond to regions contaminated by distant bright sources (Snowden et al. 1998).

We also compared our results with measurements at other wavelengths. Figure 9 shows the distribution of the local InterStellar Medium in the Galactic plane from reddening data (Lallement et al. 2014). The superimposed black line represents the contour of the LHB from our measurement which is the same as in Figure 8. The shape of the LHB matches the boundary of the local cavity very well after removing the contribution of SWCX showing that the LHB and the local cavity are closely correlated. Figure 10 shows the same data as Figure 9 but in the vertical plane and on a smaller scale. Although there is no clear boundary information of the local cavity toward high latitude, our contour matches well the local cavity at low latitude.

Based on our reconstruction of the LHB, we also calculated the total energy currently enclosed in the LHB as $3.38 \times 10^{50} \mathrm{erg}$ which is about 15.6 times smaller than estimated without removing the SWCX contribution to the RASS maps. We note that, while this is consistent with the energy released in a single supernova explosion, the LHB has been cooling away for millions of years, and its size and longevity remain inconsistent with a single SN explosion (Cox \& Anderson 1982; Cox \& Snowden 1986; Smith \& Cox 2001).

There are a few systematics which affect our results. The first is the choice of model to convert the R1 to R2 ratio to temperature, which has already been discussed, and contributes a systematic 
error of $k T=0.006 \mathrm{keV}$ to the estimate of the LHB temperature. We also investigated the effect of using different abundance tables. For example, we used the abundance table by Wilms et al. (2000) and found it has a smaller effect than the choice of model, contributing a systematic error of $k T=0.003 \mathrm{keV}$. The second major source of systematic uncertainty is the assumption made to derive the value of $\beta(t)$ by fitting the $D X L$ data, namely the choice of $\alpha_{\mathrm{H}} / \alpha_{\mathrm{He}}$ and $G$. As discussed in Uprety et al. (2016), however, although the value of $\beta(t)$ varies significantly for different combinations of $\alpha_{\mathrm{H}} / \alpha_{\mathrm{He}}$ and $G$, the total SWCX contributions are very similar. We tested different combinations and, as expected, we found that the distribution of $R 2 / R 1$ ratio after subtracting the SWCX is generally similar, and the systematic uncertainty on the peak of the distribution is $\Delta \frac{R 2}{R 1}=0.040$, corresponding a temperature difference of $k T=0.003 \mathrm{keV}$. Another possible source of systematics is the fact that the RASS R1 rate is systematically lower than other 1/ $4 \mathrm{keV}$ all-sky surveys, e.g., the University of Wisconsin sky survey (D. McCammon 2016, private communication, see also in McCammon \& Sanders 1990). Considering an $18 \%$ correction on the R1 band, to match the RASS results with previous surveys, the peak of the R2/R1 distribution would then be shifted to 0.73 , corresponding to a lower temperature of $0.088 \mathrm{keV}$.

\section{SUMMARY}

Based on the data from the $D X L$ sounding rocket mission, we quantified and removed the SWCX contribution to the foreground diffuse X-ray emission, and obtained a "cleaned" map of the LHB emission from the RASS data. Assuming that the LHB is in thermal ionization equilibrium, we measured the temperature of the LHB from the R2/R1 ratio, and estimated its emission measure over the whole sky. We found that the estimated temperature of the LHB is cooler after the contamination of the SWCX is removed. Assuming the LHB has a constant electron density, we also estimated the size of the LHB in each direction and built a three-dimensional model of the LHB, which matches quite well with maps of the local cavity from reddening data.

This work was supported by NASA award numbers NNX11AF04G and NNX09AF09G.

\section{REFERENCES}

Anders, E., \& Grevesse, N. 1989, GeCoA, 53, 197

Burrows, D. N., \& Mendenhall, J. A. 1991, Natur, 351, 629

Cox, D. P., \& Anderson, P. R. 1982, ApJ, 253, 268

Cox, D. P., \& Snowden, S. L. 1986, AdSpR, 6, 97
Cravens, T. E. 2000, ApJL, 532, L153

Cravens, T. E., Robertson, I. P., \& Snowden, S. L. 2001, JGR, 106, 24883

Crowder, S. G., Barger, K. A., Brandl, D. E., et al. 2012, ApJ, 758, 143

Galeazzi, M., Chiao, M., Collier, M. R., et al. 2011, ExA, 32, 83

Galeazzi, M., Chiao, M., Collier, M. R., et al. 2014, Natur, 512, 171

Galeazzi, M., Collier, M. R., Cravens, T., et al. 2012, AN, 333, 383

Galeazzi, M., Gupta, A., Covey, K., \& Ursino, E. 2007, ApJ, 658, 1081

Gupta, A., Galeazzi, M., Koutroumpa, D., Smith, R., \& Lallement, R. 2009, ApJ, 707, 644

Henley, D. B., \& Shelton, R. L. 2008, ApJ, 676, 335

Henley, D. B., Shelton, R. L., Cumbee, R. S., \& Stancil, P. C. 2015, ApJ, 799, 117

Jenkins, E. B. 2009, SSRv, 143, 205

Jenkins, E. B., \& Meloy, D. A. 1974, ApJL, 193, L121

Juda, M., Bloch, J. J., Edwards, B. C., et al. 1991, ApJ, 367, 182

Kaastra, J. S., \& Mewe, R. 1993, A\&AS, 97, 443

Knapp, G. R. 1975, AJ, 80, 111

Koutroumpa, D., Lallement, R., Kharchenko, V., et al. 2006, A\&A, 460, 289

Koutroumpa, D., Lallement, R., Raymond, J. C., \& Kharchenko, V. 2009, ApJ, 696, 1517

Kuntz, K. D., Collado-Vega, Y. M., Collier, M. R., et al. 2015, ApJ, 808, 143

Kuntz, K. D., \& Snowden, S. L. 2000, ApJ, 543, 195

Lallement, R. 2004, A\&A, 418, 143

Lallement, R., Bertaux, J. L., \& Dalaudier, F. 1985a, A\&A, 150, 2

Lallement, R., Bertaux, J. L., \& Kurt, V. G. 1985b, JGR, 90, 1413

Lallement, R., Vergely, J.-L., Valette, B., et al. 2014, A\&A, 561, A91

Liu, W., Galeazzi, M., \& Ursino, E. 2016, ApJ, 816, 82

McCammon, D., \& Sanders, W. T. 1990, ARA\&A, 28, 657

Mewe, R., Gronenschild, E. H. B. M., \& van den Oord, G. H. J. 1985, A\&AS, 62, 197

Michels, J. G., Raymond, J. C., Bertaux, J. L., et al. 2002, ApJ, 568, 385

Raymond, J. C., \& Smith, B. W. 1977, ApJS, 35, 419

Robertson, I. P., \& Cravens, T. E. 2003, JGRA, 108, 8031

Sanders, W. T., Kraushaar, W. L., Nousek, J. A., \& Fried, P. M. 1977, ApJL, 217, L87

Savage, B. D., \& Jenkins, E. B. 1972, ApJ, 172, 491

Smith, R. K., Bautz, M. W., Edgar, R. J., et al. 2007, PASJ, 59, 141

Smith, R. K., Brickhouse, N. S., Liedahl, D. A., \& Raymond, J. C. 2001, ApJL, 556, L91

Smith, R. K., \& Cox, D. P. 2001, ApJS, 134, 283

Snowden, S. L., Chiao, M., Collier, M. R., et al. 2014, ApJL, 791, L14

Snowden, S. L., Collier, M. R., Cravens, T., et al. 2009, ApJ, 691, 372

Snowden, S. L., Cox, D. P., McCammon, D., \& Sanders, W. T. 1990, ApJ, 354,211

Snowden, S. L., Egger, R., Finkbeiner, D. P., Freyberg, M. J., \& Plucinsky, P. P. 1998, ApJ, 493, 715

Snowden, S. L., Egger, R., Freyberg, M. J., et al. 1997, ApJ, 485, 125

Snowden, S. L., Freyberg, M. J., Kuntz, K. D., \& Sanders, W. T. 2000, ApJS, 128,171

Snowden, S. L., Mebold, U., Hirth, W., Herbstmeier, U., \& Schmitt, J. H. M. 1991, Sci, 252, 1529

Tanaka, Y., \& Bleeker, J. A. M. 1977, SSRv, 20, 815

Thomas, N. E., Carter, J. A., Chiao, M. P., et al. 2013, Proc. SPIE, 8859, $88590 \mathrm{Z}$

Uprety, Y., Chiao, M., Collier, M. R., et al. 2016, ApJ, 829, 83

Ursino, E., Galeazzi, M., \& Liu, W. 2016, ApJ, 816, 33

Wilms, J., Allen, A., \& McCray, R. 2000, ApJ, 542, 914

Yoshino, T., Mitsuda, K., Yamasaki, N. Y., et al. 2009, PASJ, 61, 805 